\title{
Les décanteurs centrifuges statiques pour le dessablage des eaux chargées
}

PAR

\author{
E. Condolios \\ Ingénieur chef du Grompe TST a Alsthom \\ Techniques des Fluides
}

\section{Introduction}

Les problèmes de double phase liquide-solide, que l'on rencontre à grande échelle dans les fleuves et les rivières, sont des problèmes auxquels se confrontent la plupart des industries, soit pour le traitement des produits qu'elles élaborent, soit dans le rejet de leurs eaux polluées.

Le liquide est alors moyen pour transporter les solides, les disperser, afin de réaliser des réactions chimiques, les classer par granulométrie ou densité. Après toutes opérations, il est nécessaire de séparer le liquide des solides afin de récupérer les deux phases.

Si la séparation d'un liquide comportant des particules solides de taille importante est relativement facile, cette opération devient beaucoup plus difficile lorsque les particules n'ont pour dimension que quelques dizaines do microns. La décantation gravitaire est longue et nécessite de lourds investissements et les industries recherchent souvent des procédés de plus faible encombrement permettant une décantation accélérée de ces particules solides.

C'est dans ce cadre que nous présenterons deux nouveaux procédés de décantation centrifuge statique ayant fait l'objet d'études hydrauliques dans les laboratoires de SOGREAH et d'Alsthom Techniques des Fluides et qui apportent des solutions nouvelles à ce type de problèmes.

Nous présenterons successivement:

- L'Hydrocyclone, appareil étudié depuis un certain nombre d'années par de nombreux utilisateurs, mais dont de nouveaux perfectionnements élargissent les possibilités d'utilisation de ce procédé.

- Le Turbiflux, appareil centrifuge lent à surface libre qui, grâce aux écoulements tourbillonnaires d'un type nouveau quil utilise, permet de diminuer de façon sensible les surfaces des bassins de décantation classique.

\section{1 - L'hydrocyclone}

Le principe tourbillonnaire du cyclone est connu de tout temps par ses méfaits dans la nature. L'utilisation de ce principe a donné lieu à la conception d'un appareil très simple qui a été utilisé depuis fort longtemps pour le dépoussiérage de lair.

Depuis le début du siècle seulement, son utilisation a commencé à apparaître comme appareil pour la séparation des particules solides en suspension dans un liquide chargé.

Ses applications sont devenues depuis très nombreuses et ses fonctions diverses:

- Décanteur épaississeur pour le dessablage des eaux chargées ou polluées avant rejet, lavage de sables argileux, concentration de pulpes de minerais ou traitement de toutes matières fines.

- Classification hydraulique pour la coupure granulométrique de sables fins, pour l'enrichissement des phosphates, la préparation des kaolins, la classification de matières fines obtenues par procédés chimiques.

- Séparateur densimétrique en milieu dense pour lo lavage des charbons et la séparation des minerais.

Du fait que cet appareil offre ces nombreuses possibilités et du grand nombre de variables qui interviennent dans son fonctionnement, les lois qui le régissent sont mal connues et complexes.

La mauvaise adaptation hydraulique de cet appareil par certains utilisateurs a entraîné parfois des échees, l'appareil étant mal adapté pour son utilisation.

Par ailleurs, le fait que de nombreux utilisateurs cherchent à obtenir, à la fois dans un même appareil, la fonction de décanteur, de classificateur ou d'épaississeur et que les conditions d'alimentation sont variables quant au débit solide des matériaux et à sa granulométrie, on observe 
alors une instabilité du fonctionnement hydraulique préjudiciable à la bonne marche de lappareil.

Les études effectuées dans les laboratoires de SOGREAH et d'Alsthom Techniques des Fluides ont permis de mettre au point un nouveau type de cyclone qui pallie, dans une large mesure, à cette instabilité hydraulique et permet de faciliter grandement l'utilisation de l'hydrocyclone dans les industries.

Cet appareil sera présenté dans la suite de notre exposé.

\subsection{PRINCIPE}

Le principe hydraulique de lhydrocyclone, comme le montre la figure 1, est relativement simple. Le liquide chargé est injecté, par l'extérieur, tangentiellement dans la partie cylindrique supérieure du cyclone.

On provoque ainsi, dans l'appareil, un mouvement de rotation qui devient de plus en plus intense au fur et à mesure que le tourbillon se développe dans la partie conique inférieure.

Si les solides contenus dans le liquide chargé sont suffisamment gros, leur densité étant homogène et plus élevée que celle du liquide, les solides sont centrifugés sur la périphérie et entraînés vers la partie basse du cyclone. Ils sortent avec une partie du liquide à la sousverse, le liquide épuré sort par la partie haute ou surverse. Le cyclone travaille comme décanteur-épaississeur.

$\mathrm{Si}$ le liquide introduit dans le cyclone est chargé de matériaux denses et homogènes de granulométric fine et grenue, on observe alors que les éléments fins sont entrainés par le courant tourbillonnaire ascendant qui se développe, dans la partie centrale de l'appareil, concentrique au mouvement tourbillonnaire principal. Les éléments grenus centrifugés à la périphérie sortent à la sousverse.

On a alors, grâce aux écoulements hydrauliques tourbillonnaires, une séparation en deux phases, grenue et fine, des matières solides en suspension. Le cyclone dans ce cas est un classificateur granulométrique.

Si le liquide entrant dans le cyclone est chargé de solides de densités différentes et que la densité du liquide est voisine ou comprise entre celle des solides, on observe alors que les particules de faible densité sont entraînées par le tourbillon central à la surverse, les particules denses sortent à la sousverse. Les solides sont alors séparés en deux fractions de densités différentes et le cyclone est un séparateur densimétrique.

Suivant la fonction à laquelle on destine lappareil, los variables géométriques ou hydrauliques le caractérisant seront différentes.

\section{2 - PRINCIPAUX PARAMĖTRES CARACTÉRISANT UN CYCLONE}

Du fait des multiples applications possibles du cyclone dans le domaine industriel, de nombreux auteurs ont étudié et proposé des formules basées sur leurs expériences permettant de déinin et de calculer les caractéristiques des cyclones.

En fait, ces formules ne peuvent s'appliquer qu'à unc certaine gamme d'appareils de formes déterminées, ne faisant pas intervenir certains paramètres comme le débit de la sousverse, la concentration en solides à l'entrée et à la sousverse de l'appareil.

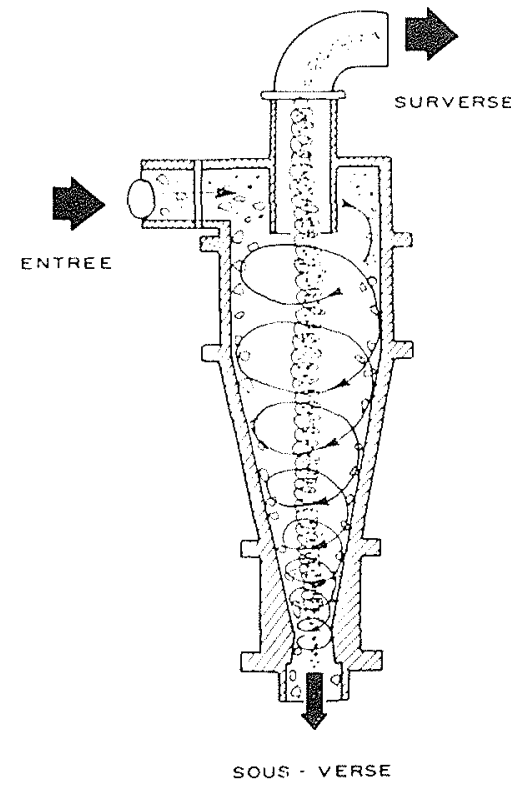

1/ Principe de tonctionnement de I'hydrocyclone.

Parmi ces formules nous citerons celles de Dahlstrom qui donnent le débit d'un cyclone en fonction de la pression d'entrée :

$$
Q=K\left(D_{1} \cdot D_{2}\right)^{1,8} \quad \sqrt{H}
$$

oì :

$Q=$ débit d'entrée du cyclone,

$H=$ pression d'entrée du cyclone,

$D_{1}=$ diamètre de la conduite d'entrée du cyclone,

$D_{2}=$ diamètre de sortie du cyclone à la surverse,

$K=$ coefficient dépendant des caractéristiques géométriques du cyclone;

et du diamètre moyen de séparation ou de décantation des particules solides de même densité en fonction du débit du cyclone:

$$
d_{501}=A \frac{\left(D_{1} \cdot D_{2}\right)^{0,6 s}}{Q^{0, j 3}} \sqrt{p_{s}-p_{c}}
$$

$d_{\%}=$ diamètre moyen de séparation à $50 \%$ de la courbe de partage,

Ps $=$ masse volumique du solide,

$P_{1:}=$ masse volumique du liquide.

Pour des domaines particuliers touchant plus spécialement l'industrie minérale, Fahlstrom a étudié, en plus des paramètres déjà cités, l'influence du diamètre extérieur du cyclone et de la section de linjection au colimaçon d'entréc, linfluence de la courbe granulométrique des solides et du débit solide à la surverse et à la sousverse.

L.es études de Fahlstrom ont mis en évidence un certain nombre de conclusions qui ont été confirmées par les études dans les laboratoires d'Alsthom Techniques des Fluides.

Une des premières conclusions est qu'un cyclone ne peut fonctionner dans de bonnes conditions que si les caractéristiques hydrauliques imposées: débit, pression d'entrée, sont compatibles avec les caractéristiques géométriques de l'appareil et les caractéristiques physiques des particules solides à séparer ou à décanter. 


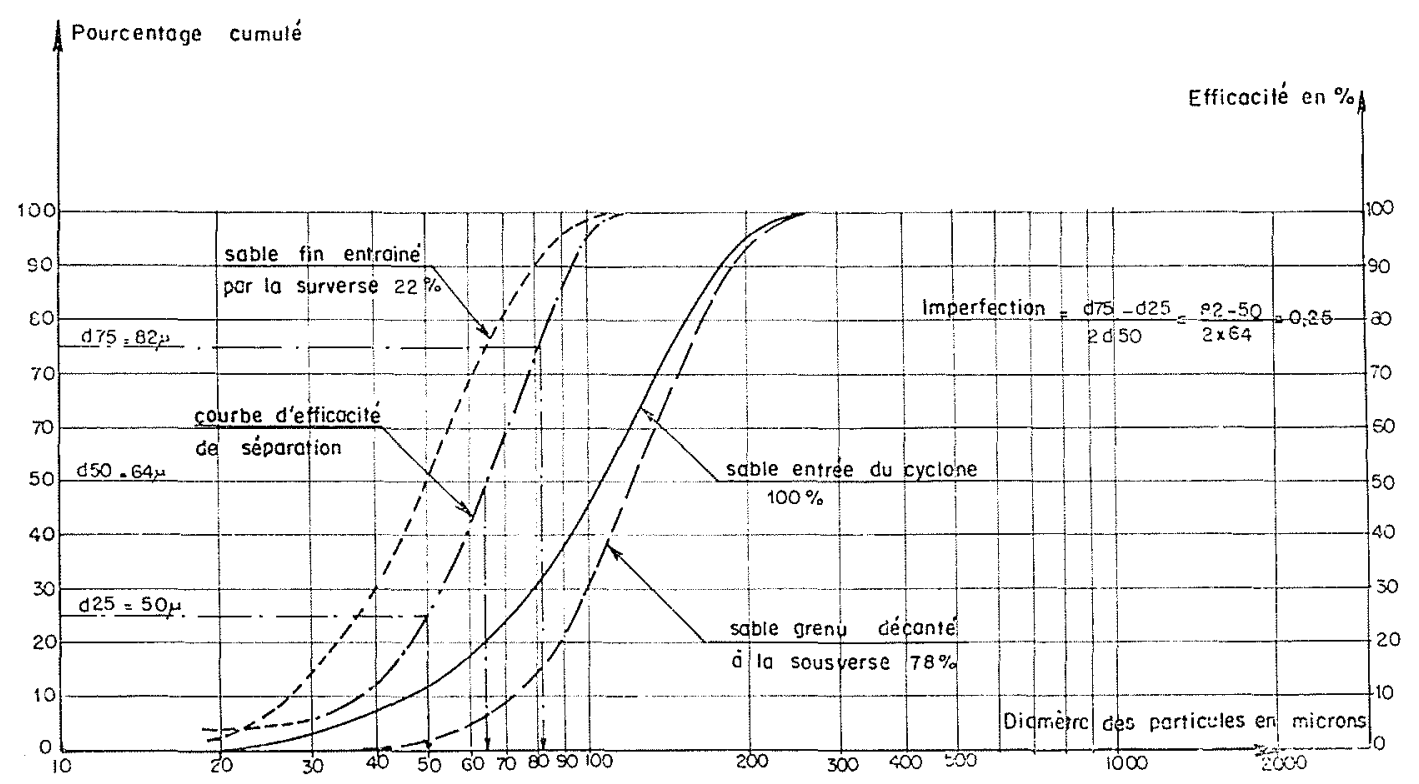

2/ Courbe d'efficacité de décantation d'un cyclone $\not 220 \mathrm{~mm}$ pour le dessablage d'eau chargée de sables fins.

Pour un cyclone déterminé, l'imperfection ou l'efficacité de séparation n'est pas indépendante du point de séparation granulométrique recherché. Elle dépend par ailleurs du rapport débit sortant à la sousverse par rapport au débit d'entrée.

L'alimentation du cyclone avec un liquide fortement chargé en solides, toutes conditions étant égales par ailleurs, entraîne une augmentation notable du point de coupure lié à la densité de la pulpe.

Un engorgement de la sousverse du cyclone, si le diamètre de la sousverse est trop petit pour le débit solide de grenus à extraire, provoque un entraînement notable de particules grenues à la surverse et une diminution importante de la qualité de séparation des particules grenues.

La figure 2 montre les courbes granulométriques: - du sable à l'entrée du cyclone (1);

- du sable grenu décanté à la sousverse du cyclone (2);

- du sable fin entrainé à la surverse (3).

Le pourcentage du sable grenu décanté est de $78 \%$, le sable fin entraîné par la surverse est de $22 \%$.

Cette figure donne également la courbe d'efficacité dc partage (4) cálcuiée à partir des courbes (1) et (2). Cette courbe montre que le diamètre moyen de séparation $d_{50}$ est de 64 microns.

Par ailleurs, la pente de cette courbe d'efficacité, comprise entre les points de partage au diamètre $d_{7 \pi}$, et $d_{25}$, permet de déterminer la qualité du pouvoir de séparation granulométrique du cyclone ou imperfection $I$ :

$$
I=\left(d_{i, 1}-d_{25}\right) / 2 d_{50}
$$

Plus la valeur de $l$ est faible, plus l'appareil est précis. Pour l'expérience présentée $I=0,25$, imperfection excellente pour ce cyclone alors que la valeur de l'imperfection se situe, pour la plupart des cyclones industriels, entre 0,30 et 40 et parfois plus pour les cyclones surchargés en matériaux ou installés sur des circuits hydrauliques mal étudiés ou instables.

\section{3 - EFFICACITÉ DE SÉPARATION D'UN CYCLONE}

Comme pour tous les appareils de décantation ou de séparation, chaque cyclone est caractérisé par une courbe d'efficacité de séparation granulométrique déduite de la courbe granulométrique des matières solides à l'entrée, des courbes granulométriques et du pourcentage d'extraction des matériaux fins et grenus sortant à la surverse et à la sousverse du cyclone.

On considere, pour un débit solide unitaire d'une tranche granulométrique entrant dans l'appareil, que ce débit se partagera et qu'un certain pourcentage sera décanté et évacué par la sousverse. Si le débit solide décanté est identique à celui de lentrée, la décantation est totale et l'efficacité de décantation de l'appareil est de $100 \%$.

Pour une tranche granulométrique plus fine, une partie seulement des matériaux sera décantée, l'efficacité de décantation aura diminué. Pour la granulométrie où la moitié du đébit solide sera décanté, l'efficacité sera seulement de $50 \%$ et ce point correspondra au diamètre moyen de séparation et au point $d_{50}$ de la courbe d'efficacité de décantation de l'appareil.

A titre d'exemple, nous donnons (fig. 2) la courbe d'efficacité d'un cyclone de $\varnothing 220 \mathrm{~mm}$ extérieur utilisé pour la décantation de sables fins en suspension dans de l'eau. Le débit d'eau chargée entrant dans le cyclone est de $13,8 \mathrm{~m}^{3} / \mathrm{h}$, le débit de sousverse est de $0,57 \mathrm{~m}^{3} / \mathrm{h}$, la concentration en sable de $100 \mathrm{~g} / \mathrm{l}$.

\section{4 - LE CYCLONE A BASCULEUR}

Comme nous l'avons dit précédemment, une des principales difficultés de l'Hydrocyclone est l'instabilité hydraulique constatée au cours de son fonctionnement, instabilité dûe à la variation du débit solide à l'entrée et du débit solide des produits grenus décantés à la sousverse.

Dans la majorité des cas d'utilisation, on cherche à 
obtenir des matériaux décantés à la sousverse aussi concentrés que possible, avec un minimum d'eau ou de liquide.

Les conditions de fonctionnement hydraulique du eyclone étant déterminées préalablement: pression d'entrée et débit fourni par la pompe d'alimentation, tracé hydraulique de la branche aval du cyclone, dimensions géométriques du cyclone et plus particulièrement de la buse de sousverse, le débit de sousverse est alors déterminé pour des cyclones classiques.

Si le débit solide à décanter est faible, le débit liquide à la sousverse étant fixé par ailleurs, la concentration en

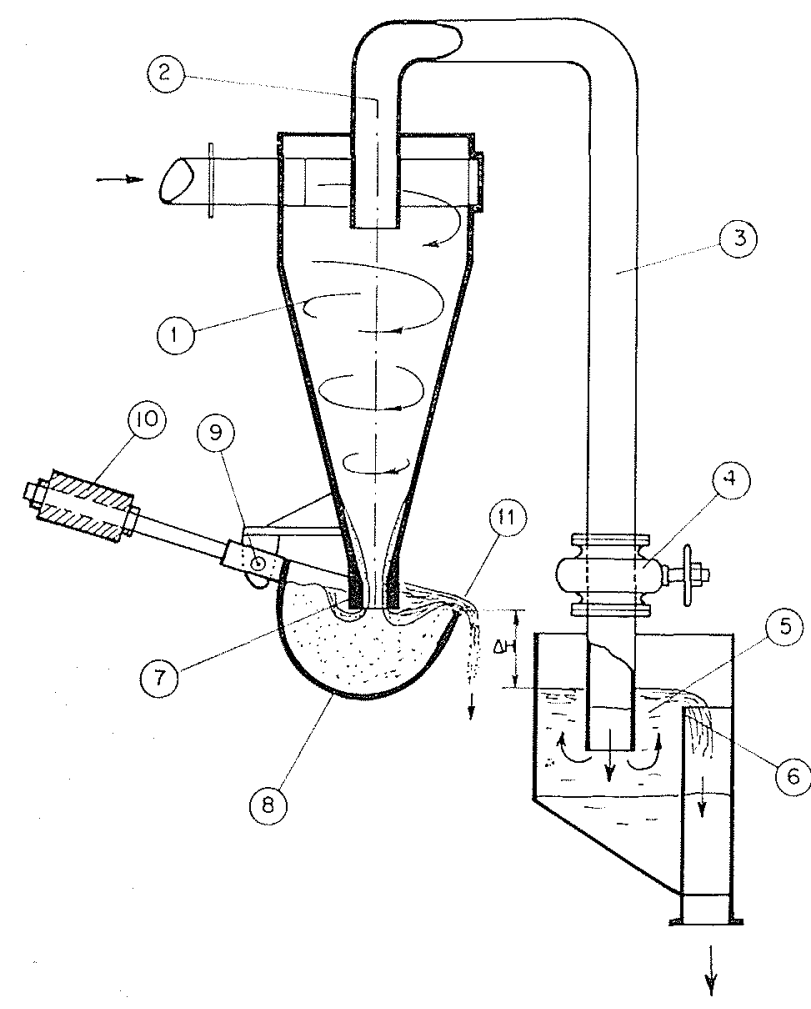

3/ Principe de fonctionnement d'un cyclone avec extracteur automatique.

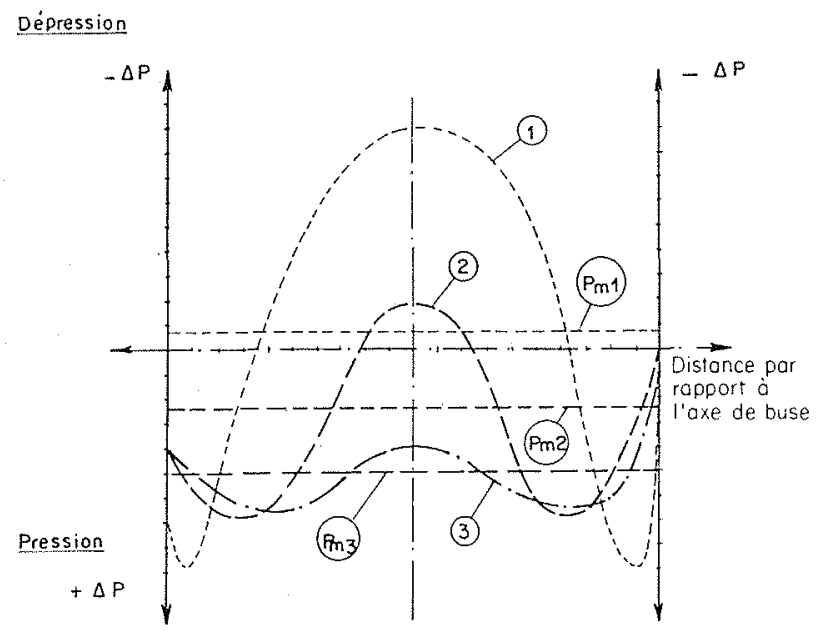

4/ Carte des courbes de pression à la buse de sousverse pour plusieurs débits solides dècantés par le cyclone. solides à la sousverse sera faible. Lorsque te débit solide augmente, les solides remplacent le liquide et la pulpe sortant à la sousverse s'épaissit pour atteindre à la limite (sables de densité 2,65), une humidité comprise entre 25 et $30 \%$ en poids/poids. Si le débit solide de sables à décanter reste constant et correspond bien au débit solide que peut extraire la buse de sousverse, le cyclone sera stable pour ce seul point de fonctionnement.

Par contre, si le débit solide à décanter dépasse les possibilités offertes par la buse de sousverse, il y aura refus à l'extraction et une partie des matériaux sera reprise par le liquide remontant à la sousverse et ne sera donc pas décantée. Avec ce type de fonctionnement, le cyclone se met à vibrer car la masse de matériaux, qui s'accumule à la pointe du cyclone, tourne d'une manière déséquilibrée et entraine de fortes perturbations dans les écoulements hydrauliques internes du cyclone.

Comme dans la grande majorité des problèmes à traitcr on note de fortes variations du débit solide de matières à décanter, il serait souhaitable que le diamètre de la buse de sousverse soit variable et s'adapte au débit solide en matériaux: la réalisation d'un tel dispositif est très difficile à obtenir dans la pratique car elle nécessite, d'une part la mesure instantanée du débit solide et, d'autre part, la commande mécanique très rapide de la section de la buse du fait du faible temps de réponse de l'appareil.

Les études hydrauliques effectuées dans nos laboratoires sur cet appareil nous ont permis d'apporter une solution nouvelle qui peut résoudre cette difficulté, grâce à l'adjonction, à la pointe du cyclone, d'un dispositif d'extraction automatique des matériaux décantés.

\subsection{1 - Principe}

La figure 3 montre ce nouveau type de cyclone. Il est constitué d'un cyclone classique (1) dont le tracé du colimaçon est prévu pour que le cyclone fonctionne avec des pressions d'alimentation comprises généralement entre 0,5 et 0,8 bar.

Le diamètre de la cheminée centrale (2) de la surverse est dans un rapport géométrique déterminé par rapport au diamètre extérieur du cyclone et à la dimension caractérisant l'entrée du colimaçon.

Par ailleurs, le diamètre de la buse de la sousverse (7) a également un rapport géométrique déterminé par rapport à la cheminée centrale.

Pour fixer les conditions hydrauliques de fonctionnement, la branche aval du cyclone (3) fonctionne en siphon, plonge dans une cuvette (5) à niveau constant et s'écoule par la crête du déversoir (6).

Le niveau du déversoir (6) est toujours calé au-dessous du niveau bas de la buse de sousverse (7).

La vanne (4), placée sur la branche aval (3), permet de régler par la variation des pertes de charge sur cette partic du circuit, la dépression moyenne à la sortie de la buse de sousverse.

Cette buse (7) plonge dans une cuillère (8) placée audessous de celle-ci. Cette cuillère est articulée sur l'axe (9) solidaire du cyclone et se trouve équilibrée par un balancier (10) de telle façon que toute variation de la pression à la pointe de la buse change la position angulaire de l'extracteur et modifie le niveau du déversoir (11) de la cuillère d'où sortent les matériaux décantés et épaissis par rapport au niveau bas de la buse (7). 
La figure 4 présente un graphique qui donne l'allure générale des courbes de pression au bas de la buse de sousverse et qui permet de comprendre le fonctionnement de l'appareil.

En effet, lorsque le cyclone fonctionne en eau sans matériaux, la courbe des pressions sur la section de la sousverse est identique à la courbe $n^{\circ} 1$. On note, du fait de l'écoulement tourbillonnaire extérieur du cyclone, une pression positive vers les parois de la buse et, du fait de l'écoulement tourbillonnaire central, une forte dépression au centre.

Cette forme de courbe montre que, dans un cylone classique, il s'échappe par la sousverse un certain débit liquide. Par ailleurs, le cyclone aspire au centre de ia buse un certain débit d'air qui vient perturber les écoulements hydrauliques tourbillonnaires internes, rend instable les pertes de charges de la branche aval, donc le débit circulant dans le cyclone.

Avec le système envisagé par la figure 3 , la buse (7) est plongée au-dessous du niveau fixé par le déversoir (11) de la cuillère (12). Le cyclone sort bien un débit d'eau à la sousverse, mais ce débit est immédiatement aspiré par la partie centrale de la buse en dépression.

Si la pression moyenne $P_{m 1}$, réglée par la vanne (4) du cyclone, est en très légère dépression par rapport à la pression zéro correspondant à la pression atmosphérique, le cyclone ne laissera pas sortir d'eau par le déversoir de la cuillère et n'aspirera pratiquement pas d'air.

Lorsqu'on introduira avec l'eau des particules solides à décanter, la pression sur la section se trouvera modifiée suivant la courbe (2) de la figure 4. Avec ce mode de fonctionnement dans un cyclone classique, les matériaux décantés prendiont en partie la place de l'eau, la pulpe extraite s'épaissira, mais le cyclone continuera à aspirer de l'air par son centre. Avec le cyclone de la figure 3, la pression moyenne $P_{m 2}$ est devenue positive. Elle agit sur la cuillère pour déplacer la position du déversoir (11) par rapport au bas de la buse. La buse continue à aspirer, du fait de la forme de la courbe de pression, l'eau sortant à la sousverse. Les matériaux décantés se déverseront de la cuillère sous une forme épaissie à humidité constante faible quel que soit le débit solide de matières traversant la buse.

Lorsque le débit solide à décanter sera tel que la courbe de pression (3), figure 4, sera en tous les points positive, la buse du cyclone sera alors engorgée et les limites d'utilisation du cyclone dépassées. Le diamètre de la buse devia donc être adapté au débit solide maximum à décanter.

\subsection{2 - Avantages du procédé}

Ce nouveau type de cyclone avec extracteur automatique des solides, dont la photo de la figure 5 montre une réalisation industrielle, offre de nombreux avantages:

- très grande stabilité hydraulique de fonctionnement, améliorant considérablement l'imperfection de séparation $I=0,25$ pour ce type de cyclone, $I=0,30$ à 0,40 pour les cyclones du type classique;

- plus faible consommation d'énergie puisque sa plage usuelle de fonctionnement est comprise entre 0,5 et 0,8 bar, alors que les cyclones classiques fonctionnent généralement entre 1,5 et 3 bars;

- aucune perte de liquide à la sousverse lorsque le débit solide de sable à décanter est nul, alors que pour les cyclones classiques ce débit est généralement compris entre 10 et $20 \%$ du débit d'entrée;

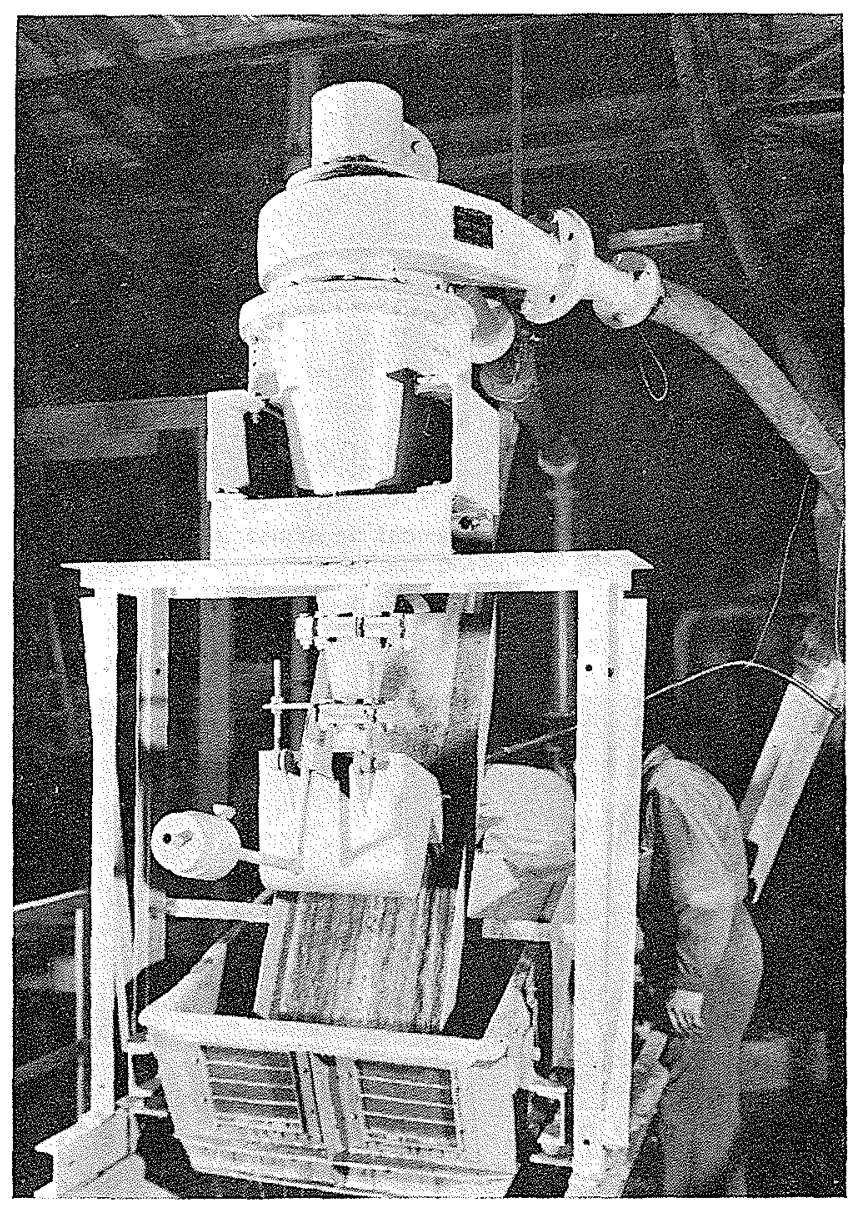

5/

- humidité des matériaux décantés constante et faible comprise entre 25 et $30 \%$ pour des sables dans de l'eau, alors qu'avec les cyclones classiques elle est généralement très élevée lorsque le débit de sable est nul et ne peut atteindre cette valeur que pour un seul point de fonctionnement;

- possibilité de mettre directement en tas les matériaux décantés et préessorés.

Tous ces avantages ont donc permis une plus grande diffusion du procédé dans tous les domaines industriels: industries minières, sablières, carrières, travaux publics, chimie, industries agro-alimentaires, rejets d'eaux industriclles, etc.

\section{5 - QUELQUES APPLICATIONS DANS L'INDUSTREE}

A titre d'exemple nous présenterons trois utilisations de ce type de cyclone:

1. Classification de sables fins industriels et récupération du kaolin.

2. Classification et lavage de phosphates.

3. Dessablage d'eaux chargées industrielles avant classilication. 


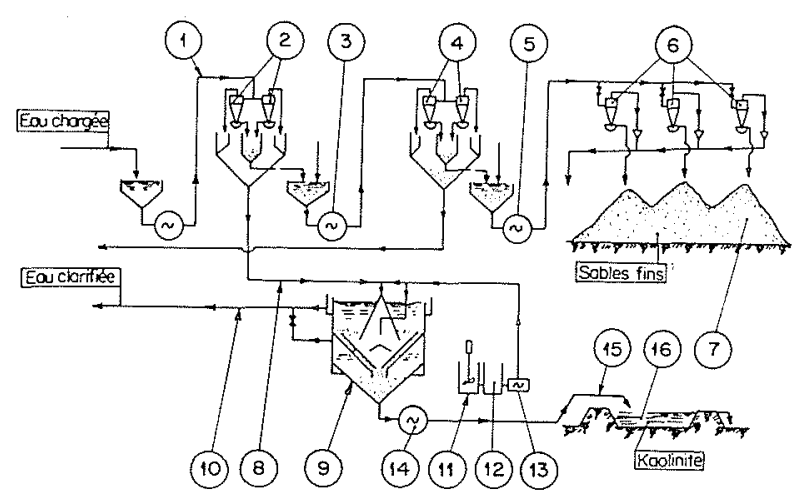

6/ Installation de traitement de sables industriels et de kaolin.

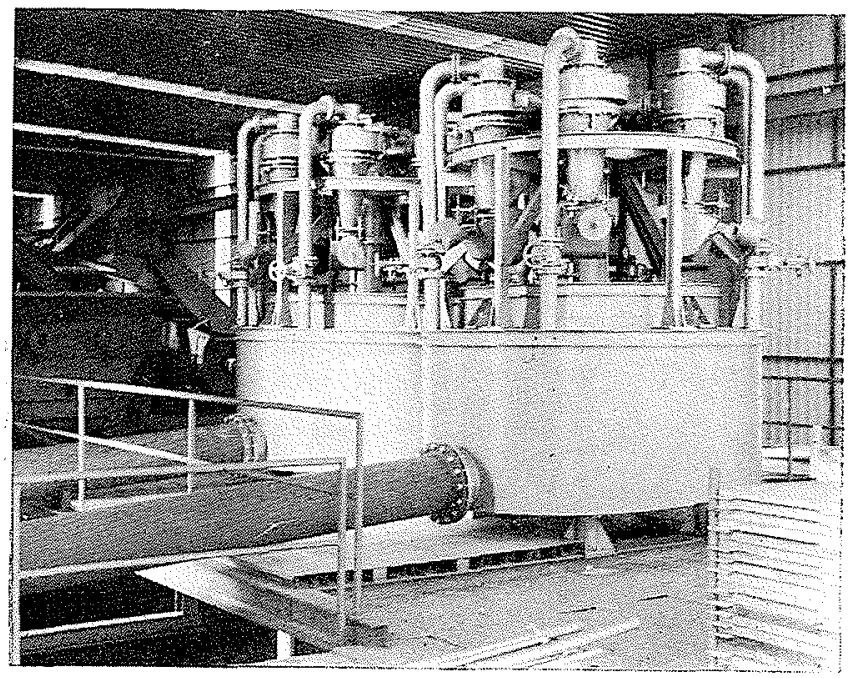

7/

\subsection{1 - Classification de sables fins}

La figure 6 montre le schéma d'une installation de traitement de sables industriels et de kaolin.

On voit sur ce schéma que l'eau chargée est envoyée sur un premier étage d'une batterie de cyclones (2) coupant à 40 microns. Les éléments fins, qui sont du kaolin, sont envoyés vers un clarificateur Seclar TPM (9), les eaux clarifiées reviennent dans l'installation, la kaolinite est envoyée vers un bassin (16) pour traitement ultérieur.

Les sables fins 40-200 microns sortant des cyclones (2) sont envoyés dans la batterie de cyclones (4) pour parfaire leur lavage. Les eaux sortant du cyclone, très peu chargées, retournent en tête de l'usine.

Les sables classés sont transportés par un transport hydraulique (5) jusqu'au hangar de stockage où se fera leur égouttage final. Les sables sont décantés par les cyclones à basculeurs (6) et mis directement en tas sur l'aire de stockage.

La photo de la figure 7 montre les deux batteries de cyclonage (2) et (4) de la figure 6 en cours de fonctionnement.

\subsection{2 - Traitement des phosphates}

La figure 8 montre l'ensemble du schéma de traitement hydraulique d'une laverie de phosphate au Maroc, traitant 3 millions de tonnes/an de phosphate.

La figure 9 montre une vue d'ensemble de la laverie.

Après débourbage (A) le minerai est classé avec de gros cyclones (2) à $0,1 \mathrm{~mm}$ au premier étage. Le minerai grenu supérieur à 0,1 est du phosphate lavé qui sera essoré définitivement sur centrifugeuse avant d'être stocké.

Le minerai fin sortant des cyclones (C) est envoyé sur un deuxième étage (D) qui élimine les éléments fins stériles inférieurs à 40 microns composés essentiellement d'argile et de silice.

La tranche granulométrique classée, comprise entre 40 et 100 microns, est envoyée dans un trieur densimétrique

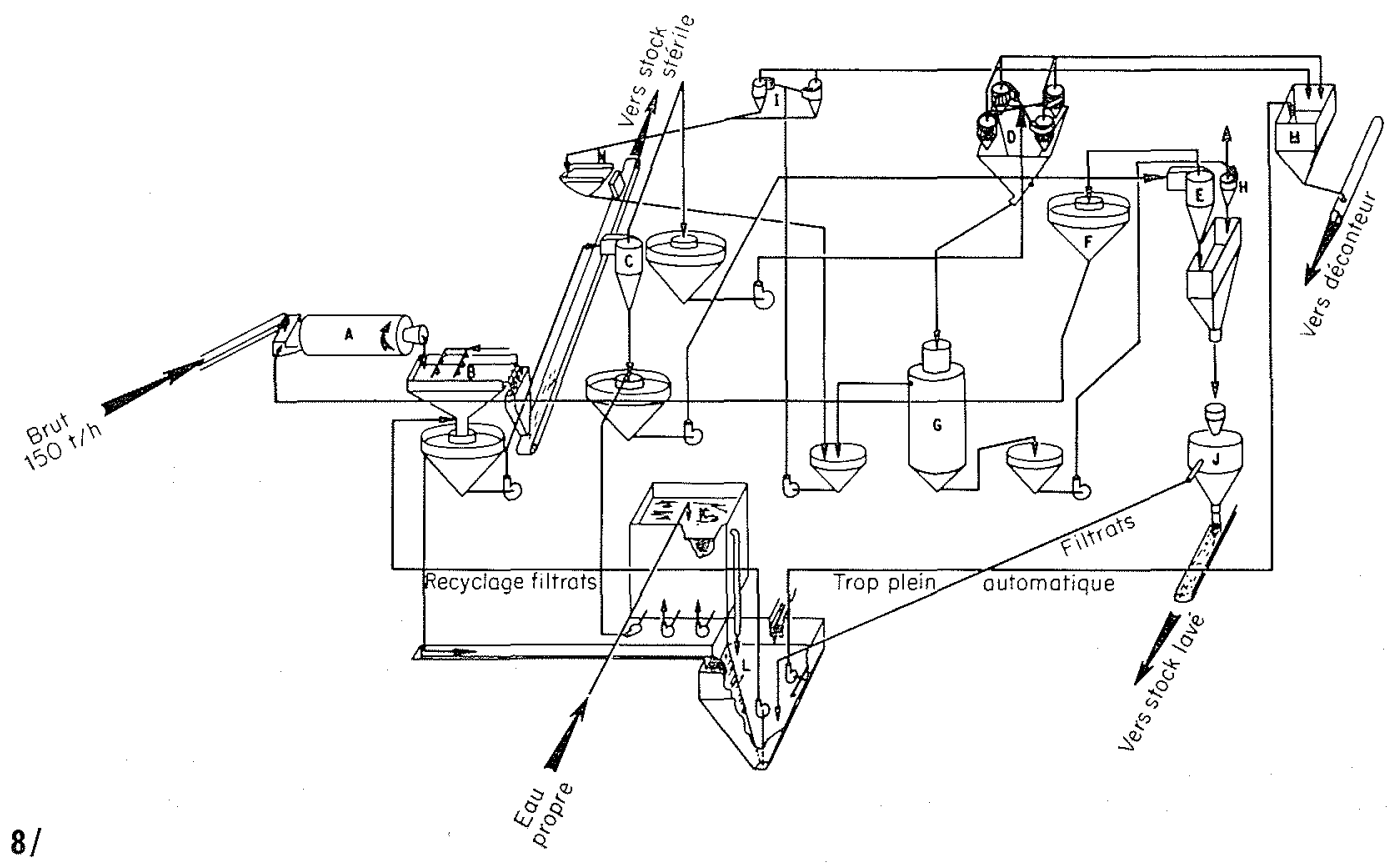


E. CONDOLIOS
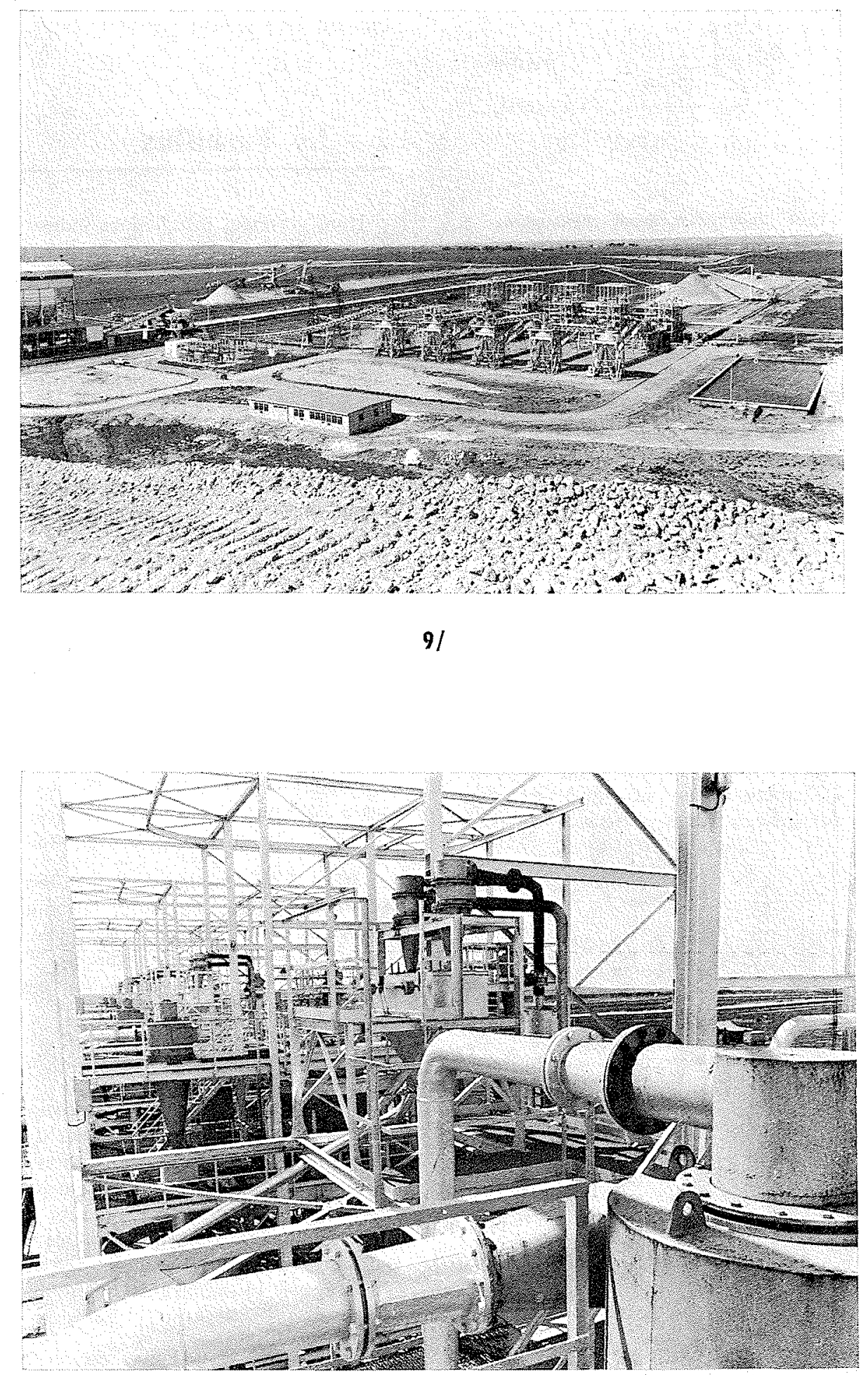

$10 /$ 


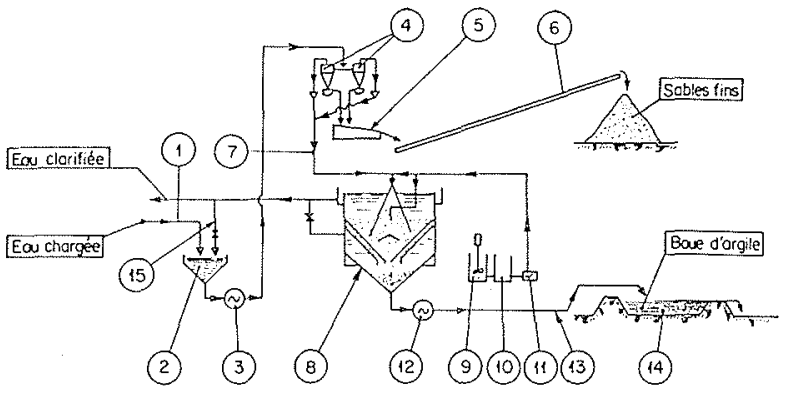

$11 /$ Dessablage d'eatx inciustrielles avant clarification.

Lavofux (G) qui sépare le phosphate et le sable siliceux qui composent cette tranche.

Le phosphate décanté par le cyclone $(\mathrm{H})$ rejoint les phosphates lavés du cyclone (E).

L.es sables fins sont décantés par le cyclone (I) et essorés avant d'être mis sur bandes et rejetés au terril.

La photo de la figure 10 montre une vue d'ensemble des cyclones d'un mètre de diamètre du premier étage.

\subsection{3 - Dessablage d'eaux industrielles chargées avant rejet}

La figure 11 montre le schéma de traitement d'eaux industrielles chargées provenant d'agrégats de carrières.

Ces eaux de lavage contiennent des argiles et des sables fins de broyage et leur pourcentage en sable peut varier suivant la marche ou l'arrêt des concasseurs secondaires.

Les eaux chargées sont cyclonées par le groupe de cyclones (2). Les sables fins récupérés, d'un diamètre compris entre 40 et 200 microns, sont stockés à terre pour être égouttés et rendus avec d'autres matériaux de construction.

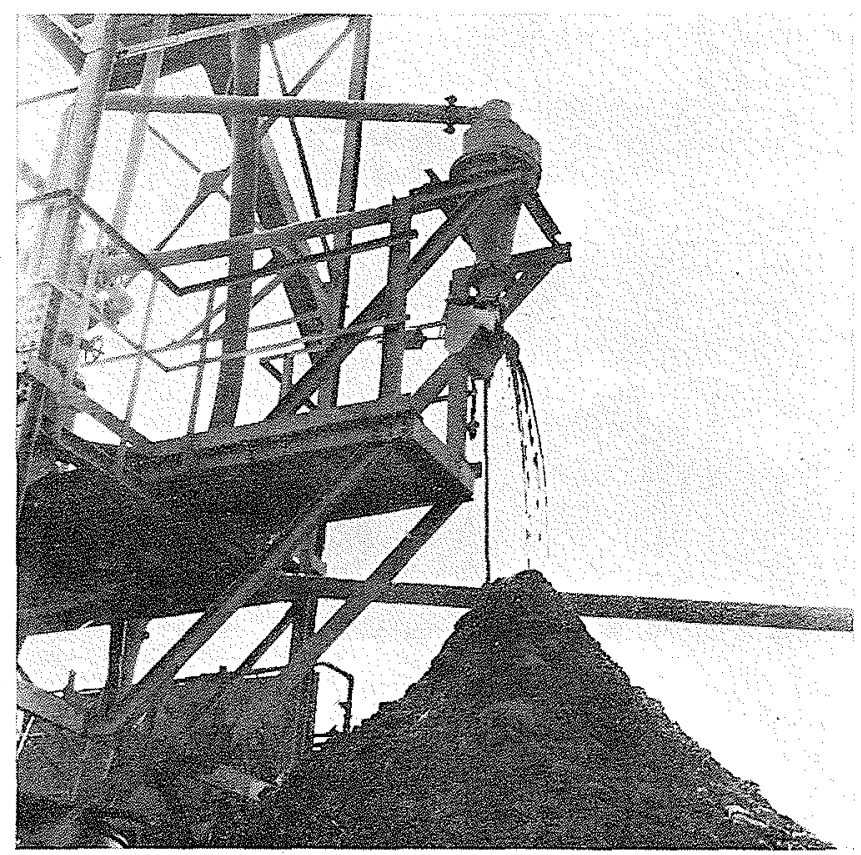

121
Les eaux sortant du cyclone sont clarifiées dans un Seclar TPM (8) avant rejet dans les eaux du domaine public, les boues argileuses sont stockées dans le bassin de stockage (10).

La figure 12 montre un des cyclones (2) de l'installation mettant directement en tas le sable fin récupéré.

\section{2 - Le Turbiflux}

Dans certaines installations industrielles qui doivent véhiculer des débits d'eaux et de solides trop importants, les cyclones, appareils qui utilisent une énergie non négligeablc, sont parfois mal adaptés pour effectuer la décantation. D'autre part, les bassins classiques à décantation naturelle par gravité occupent tellement de place et présentent de telles difficultés pour l'extraction des solides clécantés, que des recherches ont été entreprises pour résoudre ce problème particulier qui se posait avec beaucoup d'acuité pour le dragage hydraulique de sables très fins.

Des études entreprises dans les laboratoires de SOGREAH et Alsthom Techniques des Fluides, en collaboration aves la firme hollandaise Bergmann, ont permis la mise au point d'un nouvel appareil appelé Turbiflux.

Cet appareil permet, par des écoulements tourbillonnaires contrôlés, de réaliser suivant les besoins industriels recherchés :

- la séparation des particules en deux fractions, la fraction grenue extraite à la partie basse de l'appareil, la fraction fine évacuée à la partie haute;

- le lavage de la fraction grenue grâce à l'envoi dans l'appareil d'un liquide propre;

- la décantation des particules grossières dans la chambre alvéolaire inférieure de l'appareil.

Grâce à la conjugaison des différents écoulements tourbillonnaires dans l'appareil, l'action de décantation des matériaux est nettement accélérée, ce qui permet, en comparaison à d'autres appareils, d'obtenir le même effet avec des surfaces de décantation plus faibles.

\section{1 - PRINCIPE}

Le Turbiflux, décanteur centrifuge lent, a un fonctionnement hydraulique très différent du cyclone.

Dans cet appareil, la décantation n'est pratiquement pas accélérée par l'effet des forces centrifuges, mais par la forme nouvelle des écoulements qui sont provoqués. Elle permet d'obtenir une accélération notable de la décantation des particules grenues d'une dimension supérieure à 40 microns.

La figure 13 montre le fonctionnement hydraulique de l'appareil.

Cet appareil est constitué essentiellement :

- d'une chambre cylindrique(1) dans laquelle est créé l'écoulement tourbillonnaire principal dont l'intensité pourra être réglée suivant l'effet désiré;

- d'une chambre supérieure (2) cylindro-conique se rétrécissant vers la partie centrale de l'appareil à surface libre;

- dune chambre inférieure (3) conique pouvant comporter un ou plusieurs points d'extraction. 
Sans entrer dans le détail, il peut être noté que l'écoulement centrifuge engendré dans la partie centrale, grâce à un dispositif de pales statiques ou mobiles conjugué aux écoulements radiaux qui se développent dans les parties inférieure et supérieure, permet d'accélérer la décantation.

L'amélioration de la décantation est surtout due à l'allongement des lignes d'écoulements hydrauliques. Cette propriété est confirmée par les résultats obtenus avec des appareils de grandes dimensions pour lesquels on constate une amélioration notable de la décantation lorsqu'on augmente la taille de l'appareil.

Pour chaque chambre il est prévu une fonction particulière :

- dans la chambre (1) s'effectuent, grâce à des écoulements tourbillonnaires superposés par interpénétration d'un courant de liquide propre, le lavage et la pré-séparation des matériaux à séparer;

- dans la chambre (2), du fait des écoulements créés, on parfait la séparation de façon à ne laisser partir par la surverse, avec le liquide, que les éléments fins, les particules qui se décantent dans cette chambre reviennent vers la chambre (1);

- dans la chambre (3) s'effectue la décantation des matériaux grenus, les éléments fins entrainés vers cette chambre remontent par les écoulements secondaires vers la chambre (1).

Comme le montre la figure 13, le matériau dont on veut faire le lavage, la séparation ou la décantation, arrive par la conduite (10) au centre de l'appareil dans la chambre (1).

L'écoulement liquide chargé en solides pénètre dans la roue à aubes (5) dont les pales, placées de façon judicieuse, créent le mouvement tourbillonnaire central qui se propage dans l'ensemble de la chambre(1). Cette roue peut être statique, mais dans la disposition représentée dans la figure, elle est animée d'un mouvement de rotation qui est conné par l'écoulement arrivant par la conduite (10).

Le mouvement de rotation de la roue à aubes (6) qui reçoit le liquide clair de lavage est identique à celui de la roue (5) puisque les deux roues sont solidaires et montées sur le même arbre de rotation (7) porté sur les paliers mécaniques (8).

Le mouvement tourbillonnaire principal (A) projette vers la paroi cylindrique de la chambre (1) les matières solides qui sortent de la roue à aubes (5).

En même temps, le mouvement tourbillonnaire (B), alimenté en liquide propre venant de la conduite (9), permet d'améliorer le lavage des matières grenues qui décantent vers la chambre (3). Une vanne (16), placée sur la conduite (9), permet le réglage de la quantité de liquide propr: injectée dans la chambre (1).

Les matériaux grenus débarrassés de la plus grande partie des particules fines sont entraînés par le courant (B) qui circule dans la chambre(3) et se décantent dans cette chambre.

Lorsqu'on désire effectuer une simple décantation sans lavage, on peut supprimer l'arrivée de liquide propre en conservant ou non la roue à aubes (6).

Une partie du mouvement tourbillonnaire (A) qui circule sur ie plan horizontal de la chambre (1) change de direction au contact de la paroi extérieure de cette chambre, ce mouvement devient alors vertical, il traverse des orifices de forte section placés à l'extérieur de la plaque perforée (4) qui sépare la chambre (1) de la chambre (2).

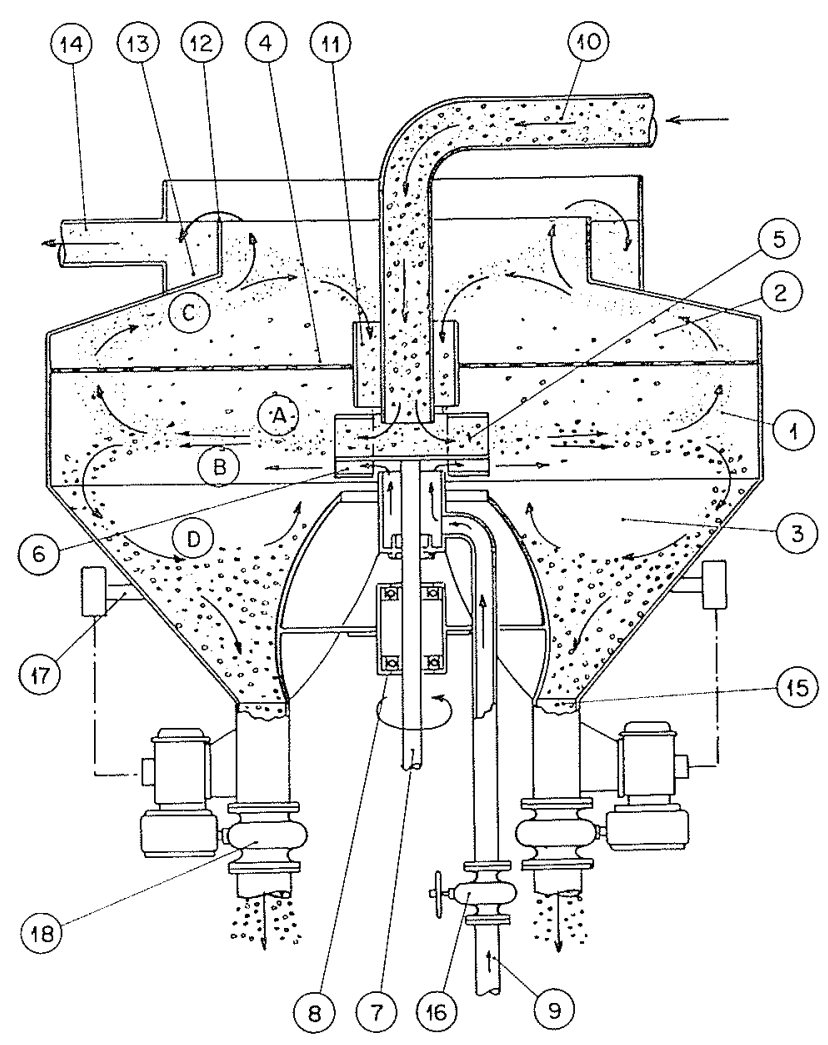

13/ Principe de fonctionnement.

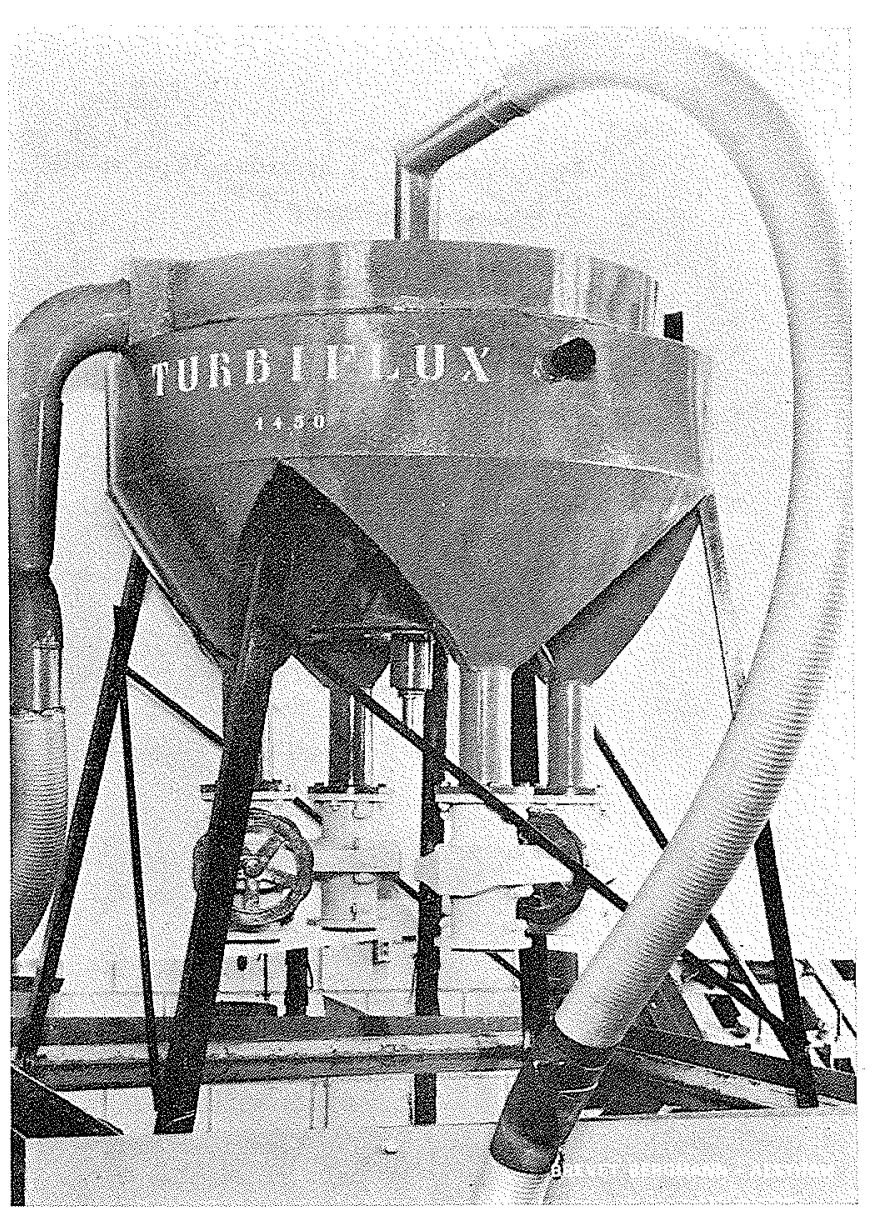

141 


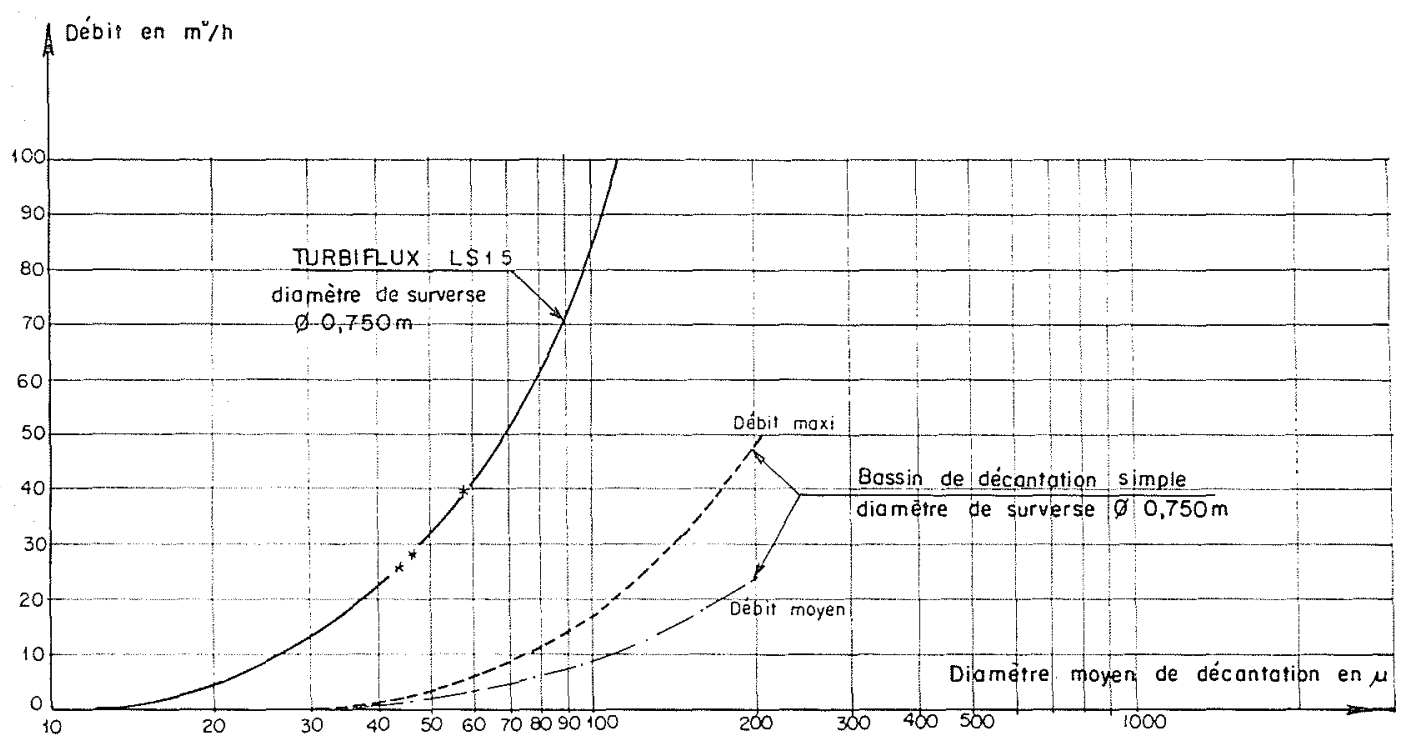

15/ Comparaison du débit en $\mathrm{m}^{3} / \mathrm{h}$ du "Turbiflux " et d'un bassin simple à débordement en fonction du diamètre moyen de décantation des particules solides.

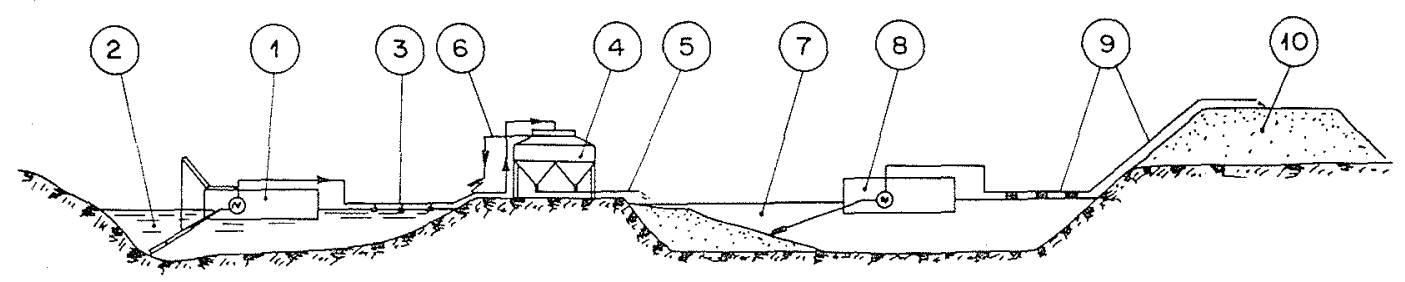

16/ Schéma d'une installation de dragage et remblayage hydraulique équipée d'un "Turbiflux LS".

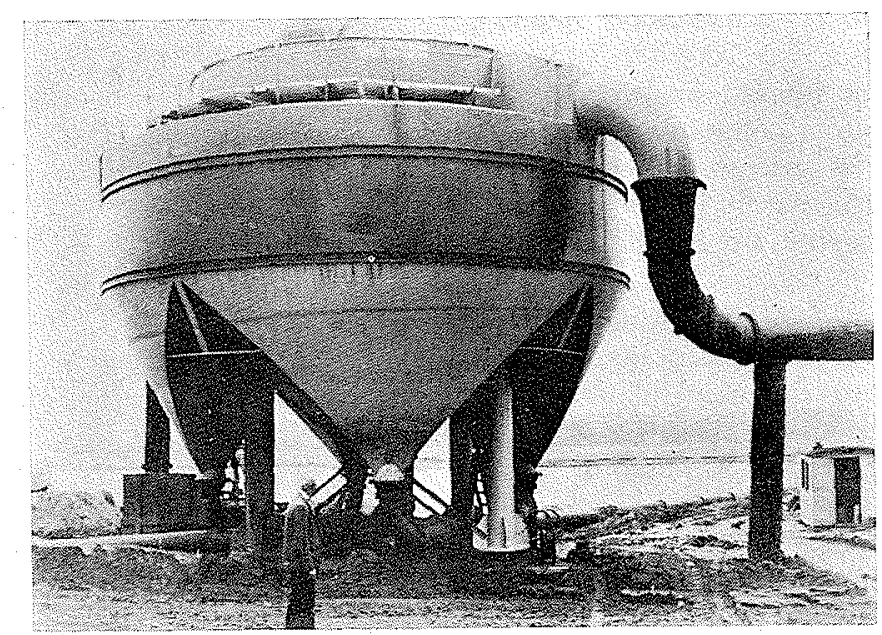

17 I 
On crée ainsi un courant tourbillonnaire (C) qui permet aux particules grenues qui auraient pu être entraînées dans le courant (C) de se décanter rapidement et de revenir; soit à travers la plaque perforée (4), soit par la cheminée centrale (11), vers la chambre (1) d'où elles pourront décanter dans la chambre (3).

Le liquide qui s'échappe par le déversoir (12) de la chambre (3) à surface libre est évacué par le collecteur (13) et la conduite (14). Ce liquide ne contient pratiquement que les particules fines à éliminer.

Des dispositions particulières peuvent être adaptées pour augmenter la concentration en solides grenus dans le liquide qui sera évacué par les orifices (15) de la chambre (3), en adaptant des détecteurs de niveau de dépôt (17) qui télécommandent, par vannes motorisées (18), le débit d'extraction des orifices (15), permettant d'obtenir des concentrations de solides élevées de l'ordre de $60 \%$ en poids/poids.

\section{2 - PRÉSENTATION DU TURBIFLUX LS 15}

La figure 14 montre une vue d'ensemble d'un appareil Turbiflux LS 15 de $1,50 \mathrm{~m}$ de diamètre.

L'appareil est équipé, au bas de la chambre de décantation inférieure, de quatre vannes en caoutchouc pour l'extraction des matériaux grenus décantés à la plus grande concentration possible.

Sur certains appareils de grandes dimensions, un palpeur permet de détecter le niveau des matériaux décantés dans les quatre trémies au bas de l'appareil et de télécommander les vannes motorisées pour l'extraction du sable décanté.

\section{3 - PERFORMANCES DE L'APPAREIL}

La figure 15 donne la courbe du point de coupure de l'appareil ( $d_{50}$ de la courbe d'imperfection) en fonction du débit de mixture entrant dans l'appareil pour la séparation des sables de densité 2,65 dans de l'eau.

L'appareil Turbiflux LS 15, montré par la figure 14, est capable de recevoir un débit moyen de mixture de $60 \mathrm{~m}^{3 /} / \mathrm{h}$ contenant $40 \mathrm{t} / \mathrm{h}$ de sable et d'effectuer une séparation à un diamètre moyen $d_{50}$ à 80 microns.

La valeur de l'imperfection réduite de la courbe d'efficacité de l'appareil est de 0,40 .

A titre de comparaison, un bassin de décantation simple d'un même diamètre extérieur au débordement que le plus grand diamètre de l'appareil, soit $\varnothing=1,5 \mathrm{~m}$, ne pourrait recevoir pour le même point de coupure qu'un débit de $25 \mathrm{~m}^{3} / \mathrm{h}$ et pour le même diamètre de débordement de l'appareil, soit $\varnothing 0,75 \mathrm{~m}$, qu'un débit maxi de $12 \mathrm{~m}^{3} / \mathrm{h}$ seulement et $6 \mathrm{~m}^{3}$ en moyenne. De telles performances sont très appréciables pour des installations importantes car elles permettent de diminuer les surfaces des bassins de décanta- tion de près de cing fois par rapport à la plus grande dimension des appareils et de dix fois sur les surfaces de surverse.

\section{4 - APPLICATION INDUSTRIELLE A L'INDUSTRIE}

Nous citerons à titre d'exemple, pour cet appareil, une réalisation importante dans le domaine du dragage.

La figure 16 montre le schéma d'ensemble de cette installation industrielle.

Une drague (1) reprend du sable fin 0-400 microns contenant $30 \%$ d'argile à éliminer. La drague refoule par conduite flottante jusqu'au Turbiflux (6) qui élimine par sa surverse les eaux argileuses, les sables vont dans un bassin (7) avec stockage sous eaux. Les mottes d'argile, qui n'ont pu être délitées au premier passage dans la pompe, se délitent par leur séjour dans le bassin.

Ces sables sont repris par la drague (8) qui refoule par la conduite (9) jusqu'au remblai (10). Ce remblai sert à l'embase d'une autoroute. La qualité du sable exigée doit contenir moins de 2 à $3 \%$ d'argile et de fines inférieures à 80 microns.

La figure 17 montre l'appareil industriel utilisé sur le chantier: un Turbiffux de $9,25 \mathrm{~m}$ de diamètre dans sa partie la plus grande et de $4,60 \mathrm{~m}$ à la surverse.

L'appareil reçoit un débit total de $5400 \mathrm{~m}^{3} / \mathrm{h}$ d'eaux chargées et d'eaux claires de lavage, avec un tonnage de sable et d'argile de $1650 \mathrm{t} / \mathrm{h}$.

Le Turbiflux renvoie dans le bassin de décantation secondaire un débit de $2000 \mathrm{~m}^{3 / \mathrm{h}}$ avec $1200 \mathrm{t} / \mathrm{h}$ de sables et rejette un débit de $3400 \mathrm{~m}^{3} / \mathrm{h}$ d'eaux argileuses contenant $450 \mathrm{t} / \mathrm{h}$ d'argile.

Le taux moyen de récupération de sable grenu est de $78 \%$. Le diamètre moyen de séparation de la courbe d'imperfection réduite est de 85 microns.

Cet appareil remplace avantageusement cinq bassins de décantation classique de $9 \mathrm{~m}$ de diamètre et de $12 \mathrm{~m}$ de hauteur et permet par ailleurs un meilleur lavage du sable.

\section{Conclusion}

La connaissance de plus en plus approfondie des lois hydrauliques régissant le système biphasique liquide-solide, tant sur le transport des matériaux en suspension ou en saltation que leur sédimentation en milieu libre ou entravé, a permis la mise au point de nouveaux procédés hydrauliques utiles à l'industrie.

Les connaissances acquises dans ce domaine apportent ainsi, dans une certaine mesure, une contribution nouvelle à l'hydraulique appliquée au domaine industriel. 


\section{Discussion}

Président: M. P. BERGeron

M. le Président remercie M. Condotios de son intéressant exposé et ouvre la discussion en présentant les remarques suivantes:

Bien qu'étant un profane dans ce domaine, je suis resté sur ma faim du fait que vous navez guère insisté sur les «écoulements secondaires $\gg$ qui me paraissent essentiels pour l'efficacité du cyclone. Très schématiquement, vous imposez à l'eau un écoulement circulairc; les particules solides - plus lourdes que l'eau - en suspension sont entrainées vers la paroi par la force centrifuge et malgré le frottement doivent tomber vers le bas de l'appareil grâce à l'existence d'un écoulement axial périphérique qui coexiste avec un écoulement axial central mais de sens contraire, c'est-à-dire, dirigé vers le haut du cyclone. M. le professeur Fortier nous a exposé, ce matim, les lois du mouvement pour qu'une particule solide soit entraînée par un liquide. Mais, dans le cyclone, il faut au contraire que la particule solide échappe à la trajectoire de l'eau qui l'entoure pour ylisser par rapport à leat vers les zones de forte pression existant dans l'appareil.

J'ai parlé, ce malin surtout, précise M. le Professeur Former, du cas où les particules solides sont extrêmement petites: de ce fait, clles peuvent très bien suivre le mouvement désordonné du liquide dô à la turbulence, mais en moyenne la vitesse de ces particules - la vitesse radiale - est différente de celle du fluide Le mouvement désordonné du liquide a des vitesses très grandes par rapport à ces vitesses moyennes radiales, et dans l'ensemble la particule progresse vers la paroi si elle a une masse volumique plus forte que celle de l'eau.

Il faut nécessairement qu'il y ait un débit de fluide qui lui-même progresse vers laxe. Les très fines particules peuvent très bien suivre ies mouvements désordonnés et échapper tout de même au fluide.

D'alute part, je voudrais demander à $M$. Condolios - dont lexposé ne comporte que quelques simples formules empiriques -si les spécialistes de SOGREAH n'ont pas tenté d'interpréter les résultats obtenus sur les cyclones par des calculs utilisant les théories récentes sur les écoulements turbulents?

Nous avons toujours commencé nos études par des essais de caractère théorique, répond $M$. Condollos, mais, très vite, nous nous sommes orientés vers l'obtention d'appareils répondant aux besoins industriels. Or, que demandent les utilisateurs? Diune part, la valeur de la perte de charge globale du cyclone et son pouvoir de décantation (ou de séparation); il s'agit de réduire au minimum la consommation d'énergie de l'appareil pour un taux de décantation aussi élevé que possible (ou une séparation aussi précise que possible).

On a done effectué des essais systématiques sur des cyclones do géométric et de dimensions différentes, alimentés par de l'eau tenani en suspension des matériatux solides de granulométries diverses. A partir des résultats obtenus, nous avons essayé de comprendre lce lois générales qui régissaient le fonctionnement de ces appareils.

Les courants secondaires jouent un rôle important dans le tourbillon principal, les grains fins sont entrainés vers lextérieur et les grains plus gros vers le centre. La séparation des grains se fait à la frontière d'une zone cylindro-cônique d'un diamètre un peu inférieur à celui de la cheminée centrale.

Sur une question de $M$. le Président, M. Condolios précise :

La couche limite a peut-être une certaine influence. On a fait des études pour voir jusqu'à quelle limite les cyclones étaient capables de «séparer». Le plus petit diamètre de cyclone que nous avons expárimenté était de $30 \mathrm{~mm}$; l'appareil fonctionnait sous des pressions très élevées.

En pratique, on a beaucoup de difficulté à décanter des particules inférieures à 5 microns de densité 2,65 en suspension dans l'eau.

Je pense, qu'à partir d'un certain moment, les effets de turbulence à l'interieur du systeme sont tels que les grains de 5 microns ne peuvent plus revenir à la paroi; ils sont peut-être repris par les couches limites.

D'antre part, ce n'est pas la gravité mais bien les courants secondaires qui assurent la sortie des matières solides. Ainsi, les cyclones à air (dépoussiéreurs) pelvent être disposés «la pointe en haut», les grains de poussière de gros diamètre n'en sortiront pas moins à la pointe de l'appareil.

Je comprends fort bien, dit M. le Professeur Fortier, que le; réalisations industrielles ne peuvent atteindre les développements avancés de la théorie mais il peut être commode de se laisser guider par celle-ci au cours des études et essais. A-t-on pu préciser si le tourbillon central du cyclone est du type « vortex » ou se rapprocho d'une rotation en bloc? Quel est l'ordre de grandeur des vitesses des différents mouvements secondaires observés?

\section{Condolios répond:}

1. Dans le cyclone le tourbillon est du type « vortex»;

2. Dans un cyclone à air bien proportionné, $90 \%$ du débit entrant sort par la «sur-verse » et $10 \%$ par la sous-verse.

Pour la définition des appareils industriels, avez-vous pu utiliser des lois de similitude, interroge M. Misson?

En ce qui concerne les cyclones, les lois de similitude sont à peu près connues aujourd hui, répond $\mathrm{M}$. Condolıos. A l'origine, des essais ont été faits sur diflérents appareils de diamètres compris entre 50 et $350 \mathrm{~mm}$. Les lois d'extrapolation déduites de ces essais ont permis de réaliser dans des conditions satisfaisantes des appareils d'un mètre et même $1,20 \mathrm{~m}$ de diamètre.

Pour le Turbiflux - basé essentiellement sur l'action de courants secondaires - les études ont porté d'abord sur des appareils de $40 \mathrm{~cm}$ puis de $1,60 \mathrm{~m}$ de diamètre; elles ont permis de passer aux engins de grande taille réalisés actuellement.

Dans les installations que vous avez présentées, les différents cyclones sont-ils alimentés par une même pompe, demande M. COUPRIE ?

Oui, confirme M. Condolios, et c'est Jà un point important. Pour un mème «point de coupure», plus on augmente le diamètre du cyclone, plus la pression à l'entrée - c'est-à-dirc l'énergie consommée - crôit; on a donc intérêt à installer des cyclones de plus petit diamètre fonctionnant à une pression plus basse.

L'une des grosses difficultés qui se pose dans le domaine industrie? est d'alimenter de façon équilibrée l'ensemble des cyclones en débit et en débit solide. Grâce au montage adopté, le cyclone fait sa propre régulation sur le débit «liquide-solide».

M. le Président clôt la discussion et donne la parole à M. Couratis pour l'exposé de la communication qu'il a établie en collaboration avec M. Condolios. 